\title{
Scattering on excited atmospheric components as a cause of the increase of lidar signal in the upper and middle atmosphere
}

\author{
Vasily Bychkov ${ }^{1}$, Ilya Seredkin
}

Institute of Cosmophysical Researches and Radio Wave Propagation of the Far Eastern Branch of the Russian Academy of Sciences, Paratunka, Russia

\begin{abstract}
The lidar data of the resonant scattering in the upper and middle Kamchatka atmosphere are analyzed. It is shown that the increase of the scattering signal at altitudes of $350-450 \mathrm{~km}$ at $561 \mathrm{~nm}$ may be due to the scattering of the maximum of layer F2 excited by precipitated electrons on ions. Large variations in the signal at these altitudes are caused by spatial plasma inhomogeneities in the ionosphere, as confirmed by the ionosonde data. The analysis of the interaction of a laser pulse with excited ions in the stratosphere is refined, and the effect of collisions on the lifetime is taken into account. It is shown that for the used lidar in the middle atmosphere for altitudes above $10 \mathrm{~km}$, the conditions of guaranteed interaction with the radiation of each ion born in the strobe are satisfied.
\end{abstract}

\section{Introduction}

The Kamchatka Lidar Station equipped by the Brilliant-B laser and Newton's telescope was commissioned in autumn 2007. When investigating the phenomenon of the photomultiplier effect at wavelength 532 in March 2008, a lidar signal was detected in the $200-300 \mathrm{cH} \mathrm{km}$ altitude region. It was explained as a resonant scattering signal on excited atomic nitrogen ions. Signals of nm backscatter in the region of 200-300 km are presented in [1]. The results of two-frequency sensing at wavelengths 532 and $561 \mathrm{~nm}$ in the $100-500$ $\mathrm{km}$ region are presented in [2-4]. On the basis of data from ionospheric and lidar observations it was shown that the total lidar signal at an altitude of $200-300 \mathrm{~km}$ could correlate with the plasma content in the region of the maximum F2 night layer of the ionosphere when superthermal $(0.1-10 \mathrm{keV})$ electrons precipitated into the atmosphere.

The analysis of geophysical data accompanying the phenomenon led to the conclusion that the possible physical mechanism explaining these correlations is the resonant scattering of atomic nitrogen on excited ions. The phenomenon was observed both in magnetically calm conditions and during geomagnetic disturbances.

The used equipment, methods of recording the main and background signals, methods of data processing have been repeatedly cited in our other works, for example [3-

1Corresponding author: vasily.v.bychkov@gmail.com 
5], and are not reproduced in this work. For the convenience of presentation the Table of used electronic transitions is reproduced.

Table 2. Dipole transitions of excited ions of oxygen and nitrogen atoms falling within the radiation bands of lasers [6].

\begin{tabular}{|c|c|c|c|c|c|c|c|c|c|}
\hline & $\begin{array}{c}\text { Com- } \\
\text { ponent }\end{array}$ & $\begin{array}{c}\text { Wavelength } \\
\text { Air }(\mathrm{nm})\end{array}$ & $\mathrm{A}_{\mathrm{ki}}\left(\mathrm{s}^{-1}\right)$ & $\begin{array}{c}\text { Lower } \\
\text { Level }\end{array}$ & Term & $\mathrm{J}$ & Upper Level & Term & $\mathrm{J}$ \\
\hline 1 & O II & 561.1072 & $2.14 \mathrm{e}+06$ & $2 s^{2} 2 p^{2}\left({ }^{1} \mathrm{~S}\right) 3 s$ & ${ }^{2} \mathrm{~S}$ & ${ }^{1 / 2}$ & $2 s^{2} 2 p^{2}\left({ }^{3} \mathrm{P}\right) 4 p$ & ${ }^{2} \mathrm{P}^{\circ}$ & ${ }^{1 / 2}$ \\
\hline 2 & NII & 532.0958 & $2.52 \mathrm{e}+07$ & $2 \mathrm{~s} 2 \mathrm{p}^{2}\left({ }^{4} \mathrm{P}\right) 3 \mathrm{p}$ & ${ }^{5} \mathrm{P}^{\circ}$ & 1 & $2 \mathrm{~s} 2 \mathrm{p}^{2}\left({ }^{4} \mathrm{P}\right) 3 \mathrm{~d}$ & ${ }^{5} \mathrm{P}$ & 2 \\
\hline
\end{tabular}

Here OII is an ion $\mathrm{O}^{+}$, NII - ion $\mathrm{N}^{+}, \operatorname{Ref}^{3}$. The lines represented in Table 1 were chosen on the basis of the laser radiation band widths and ion line Doppler broadening at the ionospheric heights of $\sim 0.004 \mathrm{~nm}$ for the temperature of $800^{\circ} \mathrm{K}$.

\section{Experimental data}

Between August and November 2017, seven cases of backscatter on both lidar channels were recorded. A characteristic feature of the data obtained during this period is the $200-400 \mathrm{~km}$ scattering and its absence at altitudes of 100-200 km. In all cases, the geomagnetic environment was calm during the lidar observations. Figure 1 shows the spatial and temporal distribution of the reverse signal received on 5 September 2017.
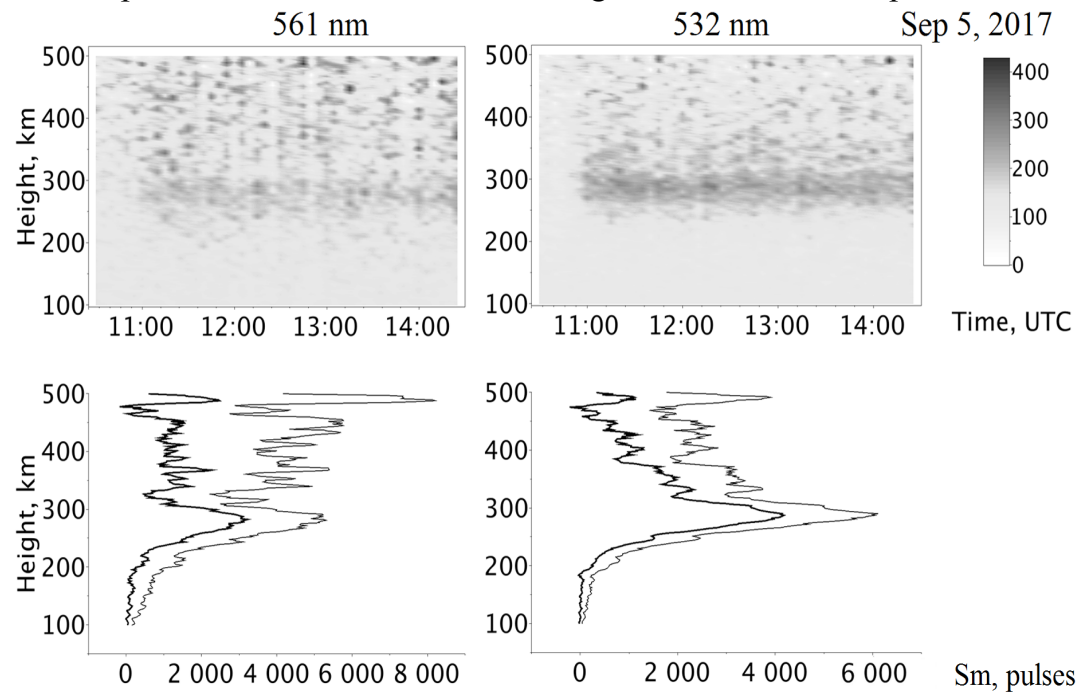

Fig. 1. Lidar "signal minus background" in the area of 100-500 km (top) and modified Sm signal (bottom) received September 5, 2017.

In the upper part of the figure lidar data with 5-minute accumulation and spatial resolution of $1.5 \mathrm{~km}$ are used. The background signal is subtracted. The profile is smoothed in height by the Moving Average method with $7.5 \mathrm{~km}$ window. The signal values are modified by multiplying the signal by the coefficient $\mathrm{k} \cdot \mathrm{H}^{2}$, where $\mathrm{H}$ is height, $\mathrm{k}=10^{-4}$. The use of 5-minute data allows to reveal in more detail the distribution in time of backscatter signals. Modifying the signal by multiplying by a square of height corresponds to the actual reduction of the real signal as the height increases. With the selected factor $\mathrm{k}=10^{-4}$ at an altitude of $100 \mathrm{~km}$, the signal matches the actually recorded signal, thus improving the visual perception of the data. Thin lines represent the standard deviation of the signal. 
The picture in black and white shows more clearly the heterogeneity of the signal at heights above $300 \mathrm{~km}$. These irregularities are more pronounced in the signal with a wavelength of $561 \mathrm{~nm}$.

\section{Discussion of results}

\subsection{Lower thermosphere}

The papers [3-5] discussed the main features of lidar sensing data obtained in $\mathrm{Au}-$ gust-November 2017. The following general features of the signal are characteristic for all events: $561 \mathrm{~nm}$;

- values of the total overnight signal at $532 \mathrm{~nm}$ were usually $20-40 \%$ higher than at

- the maximum of scattering signals is below the maximum $\mathrm{O}+$ content in layer $\mathrm{F} 2$;

- the second local maximum at $320-420 \mathrm{~km}$ is typical only for signals with $561 \mathrm{~nm}$ wavelength.

- the signal profile matches well with that of the ionisation rate profile of the mono energy flow of electrons.

Based on these features, it is concluded that the main maximum signal (Figure 1) is formed by scattering on excited ions born in the process of ionization in the excited state. The signal of scattering on the existing ions of the F2 layer excited by the precipitated electrons should be maximally displayed at the height of maximum F2 layer. Under the conditions of the events observed in autumn 2017, this area was located above $300 \mathrm{~km}$, on September 5, up to $350-400 \mathrm{~km}$ at the end of the observations. This scattering can explain the second local maximum signal at $561 \mathrm{~nm}$.

The large variations of the signal at $561 \mathrm{~nm}$ (Figure 1) in the 300-500 km range can be explained not only by the small value of the original signal in this altitude range. The study of signal profiles with 15, 30 and 60-minute accumulation showed that at the altitudes of 300-500 km there are negative values of "signal minus background", in amplitude comparable with the positive values in the regions $10-20 \mathrm{~km}$. This means the appearance of areas with values of the reverse signal below average background values and is explained by the presence of irregularities in the height distribution of plasma. As confirmed by ionospheric observations - according to the ionosonde data, a significant diffusion of the foF2 trace (F- spread) was recorded in all cases of the appearance of light-scattering layers in the ionosphere. The presence of such inhomogeneities could be seen directly in figure 1.

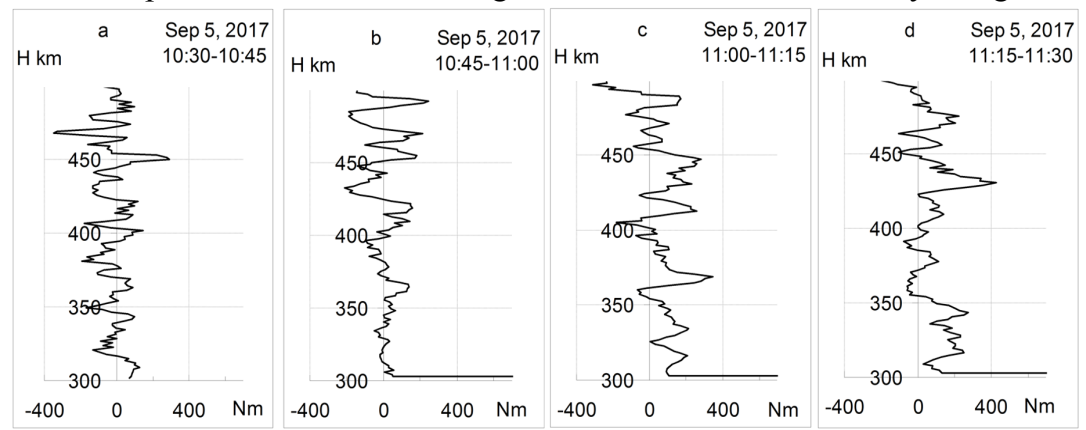

Fig. 2. Profiles of the signal with 15 -minute accumulation at $561 \mathrm{~nm}$ wavelength, received 05.09.2017 
Figure 2 shows the profiles of signals with 15-minute accumulation obtained during the first hour from the appearance of the light-scattering layer from 10:30 UTC to 11:30 UTC. The signal has been modified according to the same scheme as the data in Figure 1.

Profiles with 30-minute and hourly accumulation look similar. The summation of such signals should have given the resultant signal similar to the one shown in Figure 1. The large value of the confidence interval, 3-5 times greater than the values of the main signal, as well as the lack of any patterns in the behavior of signals with a smaller interval of accumulation does not allow us to draw any conclusions about the details of the behavior of the total signal at an altitude more than $300 \mathrm{~km}$.

\subsection{Middle Atmosphere}

As shown in [5], the conditions for the appearance of resonant scattering are preserved in the middle atmosphere. According to the NRLMSIS-00 model [6], atomic oxygen appears at altitudes of $75-80 \mathrm{~km}$, its content rapidly increases to a maximum at an altitude of $\sim 100 \mathrm{~km}\left(4 \cdot 10^{11} \mathrm{~cm}^{-3}\right)$ and then rapidly decreases on $300 \mathrm{~km}\left(5 \cdot 10^{8} \mathrm{~cm}^{-3}\right)$. Atomic nitrogen appears at altitudes of about $100 \mathrm{~km}$, its content reaches its maximum at an altitude of $200 \mathrm{~km}\left(5 \cdot 10^{7} \mathrm{~cm}^{-3}\right)$ and drops by more than an order of magnitude at an altitude of $400 \mathrm{~km}$. The absence of significant amount of atomic nitrogen and oxygen at the altitudes of the middle atmosphere does not mean that their ions do not occur there. With the appearance of ionization sources, these ions are born, often in an excited state, and die quickly in recombination processes. The main sources of their appearance can be molecular oxygen, nitrogen and ozone.

The lifetime of these ions is determined by the frequency of their collisions with neutrals, during which they are transformed into heavy ions with short lifetime. But lifetime of the investigated excited states in thermosphere and mesosphere is much shorter than life time of ions.

The collision frequency of ions with neutrals can be calculated using a formula:

$$
v(\mathrm{~N})=0.81 \cdot 10^{-10}(\mathrm{~T} / \mathrm{M})^{1 / 2} \mathrm{~N}\left(\mathrm{c}^{-1}\right) \quad(1)
$$

where $\mathrm{T}$ is temperature, $\mathrm{M}$ is molecular mass, and $\mathrm{N}$ is the concentration of atmospheric particles [6]. Lifetime of the excited ion $\tau$ is defined as:

$$
\tau=1 /\left(\Sigma \mathrm{A}_{\mathrm{ki}}+v\right)
$$

(2).

where $v$ is the impact frequency of ions with neutrals, $A_{k i}$ is Einstein's coefficients determining the probability of transition, the summation of coefficients is made for all states, transitions to which are allowed [7].

Figure 3a presents lifetime profiles $\tau$ for the investigated excited states of oxygen and nitrogen ions. Calculation of the sum of Einstein coefficients was carried out using NIST Atomic Spectra Database [6] by searching for all states, transitions to which are possible. When calculating the collision frequency of ions with neutrals, the atmospheric model NRLMSIS-00 was used [8].

Figures $3 b$, $3 c$ show the values of "additional" signal Sa, due to the sum of aerosol and resonant scattering [5]. In general, the value of molecular scattering with a known scattering ratio can be calculated as $(\mathrm{N}-\mathrm{Nf}) / \mathrm{R}$, where $\mathrm{N}$ is the total lidar signal, $\mathrm{Nf}$ is the background signal and $\mathrm{R}$ is the scattering ratio. Recall that the value $\mathrm{R}=1$ means that only the molecular scattering exists. By denoting $\mathrm{S}=(\mathrm{N}-\mathrm{Nf})$ we obtain for the additional signal $\mathrm{Sa}$ a simple expression $\mathrm{Sa}=\mathrm{S}-\mathrm{S} / \mathrm{R}$. 


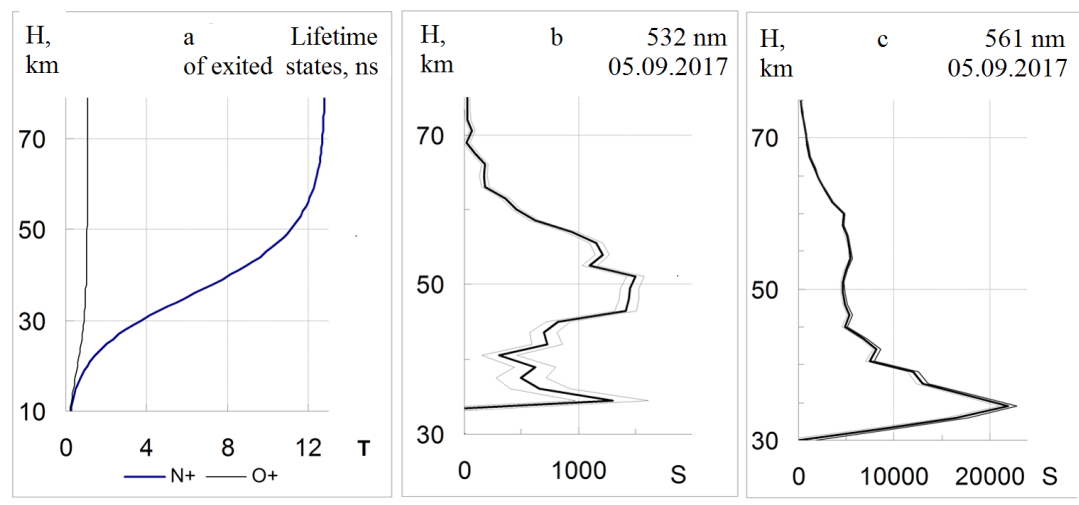

Fig. 3. Profile of lifetime of the lower level states of Table 1 in the average atmosphere (a), the total lidar signal Sa minus the "molecular scattering signal and the background signal" (b, c) received on September 5, 2017.

The estimation of electrons' spectra precipitated at the average atmosphere heights was carried out in [5]. In [9, 10], the signal values were estimated and a method for estimating the probability of the interaction of an excited atom with a photon of a laser pulse was given. The main parameter determining the probability of interaction with the radiation of the excited ion born in the strobe can be considered parameter $P=\sigma \cdot N \tau$. Here $N \tau$ is the number of photons of one pulse passing through the unit of the illuminated surface during the lifetime of the ion, $\sigma$ is the cross-section of the resonant scattering of the excited ion. In the general case, $N \tau$ depends on the pulse energy and duration, the lifetime of the excited state other parameters. If $\sigma \cdot \mathrm{N} \tau$ is greater than one, the probability of interaction is close to 1 , otherwise it is equal to $\sigma \cdot \mathrm{N} \tau$. In this case, the lidar signal $\mathrm{N}$ can be estimated by the expression [10]:

$$
\mathrm{N} \sim \mathrm{V} \cdot\left(\tau+\mathrm{T}_{\text {pulse }}\right) \cdot \mathrm{P} \quad \text { (3) }
$$

The signal must be proportional to the birth rate of excited ions $\mathrm{V}$ multiplied by the probability of interaction of the excited state with the photon of laser pulse $\mathrm{P}$ and on $\left(\tau+\mathrm{T}_{\text {pulse }}\right)$. It was shown in [10] that for the lidar used, the probability of the interaction of each excited ion born in the strobe at heights from $10 \mathrm{~km}$ and higher can be considered equal to one.

All the above considerations refer to the interaction of excited states of the "lower level" of Table 1. The influence of collisions with neutral components on the rate of appearance of these states requires a separate evaluation and experimental verification. This is explained by the fact that the excited states under study can be obtained by cascading transitions from states with higher energy level. And collisions can have a significant impact on this process.

As noted in [4] in the thermosphere, the resonance scattering signal on excited nitrogen ions is $20-40 \%$ higher than the scattering signal on excited oxygen ions. At the altitudes of the niddle atmosphere the resonance scattering signal on oxygen ions is more than one order of magnitude higher than the signal on nitrogen ions (Figures 3b, 3c). This can be explained by the presence of ozone, which has a large ionization cross section accompanied by dissociation and the appearance of excited atomic oxygen ions.

\section{Conclusion}

In the Earth's atmosphere, the main process leading to the appearance of resonant scattering on gas components of the atmosphere is ionization with the appearance of 
excited ions. Under night conditions, this is primarily the case with the ionization of precipitated electrons. In this case, the presence in the atmosphere in appreciable amounts of both ions in the ground state and neutral atoms of oxygen and nitrogen is not necessary.

The scattering of atomic oxygen and nitrogen of the F2 layer on the atomic oxygen and nitrogen ions that appeared in the process of excitation by the precipitated electrons can be observed at heights more than $300 \mathrm{~km}$ where the concentration of the main ionizable molecular components decreases. Large variations in the signal in this altitude region are caused by weakness of the signal itself on a high background and the presence of plasma inhomogeneities. Electron precipitation is usually accompanied by the phenomenon of Fspread on the inhomogeneities in the plasma distribution, which leads to additional variations in the signal profile.

At altitudes less than $300 \mathrm{~km}$, the scattering of the existing F2 layer ions that appeared during the excitation process will be masked against the background of more intense scattering on ions born during the ionization of neutral atmospheric components.

The work was performed as part of the State task on "Dynamics of physical processes in the near space active zones and geosphere" (2018-2020) № state registration AAAA-A17-1170801100434. The work was carried out by the means of the Common Use Center "North-Eastern Heliogeophysical Center" CKP_558279, USU 351757.

\section{References}

1. V.V. Bychkov, Y.A. Nepomnyashchiy, A.S. Perezhogin, B.M. Shevtsov, 28, 303-307 (2015)

2. V.V. Bychkov, I.N. Seredkin, A.S. Perezhogin, B.M. Shevtsov, V.N, Marichev, Optika atmosfery i okeana, 30, 209-213 (2017)

3. V.V. Bychkov, A.S. Perezhogin, I.N. Seredkin, B.M. Shevtsov. On the role of the method of measuring the background signal in the lidar measurements of the upper Atmosphere SPIE. Proc., 10466, 1046677 (2017)

4. V. V. Bychkov, A. S. Perezhogin, I. N. Seredkin, B. M. Shevtsov Appearance of lightscattering layers in the thermosphere of Kamchatka during the autumn of 2017, Proc. SPIE. 10833A4 (2018)

5. V. V. Bychkov, A. S. Perezhogin, I. N. Seredkin Resonant scattering as a cause for the registration of scattering layers in the thermosphere and imaginary aerosol formationsin the middle atmosphere E3S Web of Conferences 127, 01009 (2019)

6. A.Kramida, Yu.Ralchenko, J. Reader and NIST ASD TEAM. "NIST Atomic Spectra Database (ver. 5.5.2)" URL: https://physics.nist.gov/asd, National Institute of Standards and Technology, Gaithersburg, MD (2018)

7. N.N. Shchefov, A.I. Semenov, V. YU. Khomich Airglow as an indicator of the upper atmospheric structure and dynamics. Moscow, GEOS, 740 (2006)

8. M. Picone, A.E. Hedin, D. Drob NRLMSISE-00 Model 2001 URL: https://ccmc.gsfc.nasa.gov/modelweb/atmos/nrlmsise00.html

9. Bychkov V.V., Seredkin I.N., Optika Atmofery i Okeana (to be published)

10. Bychkov V.V., Seredkin I.N., Marichev V.N., Optika Atmofery i Okeana (to be published) 